\title{
New Look for Cochrane Library
}

The Cochrane library has recently updated its website focusing on its two core databases, the Cochrane Database of Systematic Reviews (CDSR) and the Cochrane Central Register of Controlled Trials (CENTRAL). (Fig. 1 Cochrane Library Home Page)

The CDSR includes all Cochrane Reviews (and protocols) prepared by Cochrane Review Groups as well as editorial and supplements. CDSR is continuously updated, with new and updated reviews and protocols being published when ready.

CENTRAL is a source of reports of randomised and quasi-randomised controlled trials published on a monthly basis. Records contain bibliographic details often including an abstract but not the full text. In addition to the two core databases the library includes Cochrane Clinical answers. These are clinically focused summaries of published Cochrane reviews. They include a clinical question, a short answer and the data deemed most relevant for practising clinicians from the Cochrane review.

Figure 2 shows the results of a search of the library using the term 'toothpaste' which identifies 22 Cochrane reviews, two Cochrane Protocols, 1697 trials and one clinical answer. The new site also lets you filter your results by date, language and Cochrane Topic (Review Group).

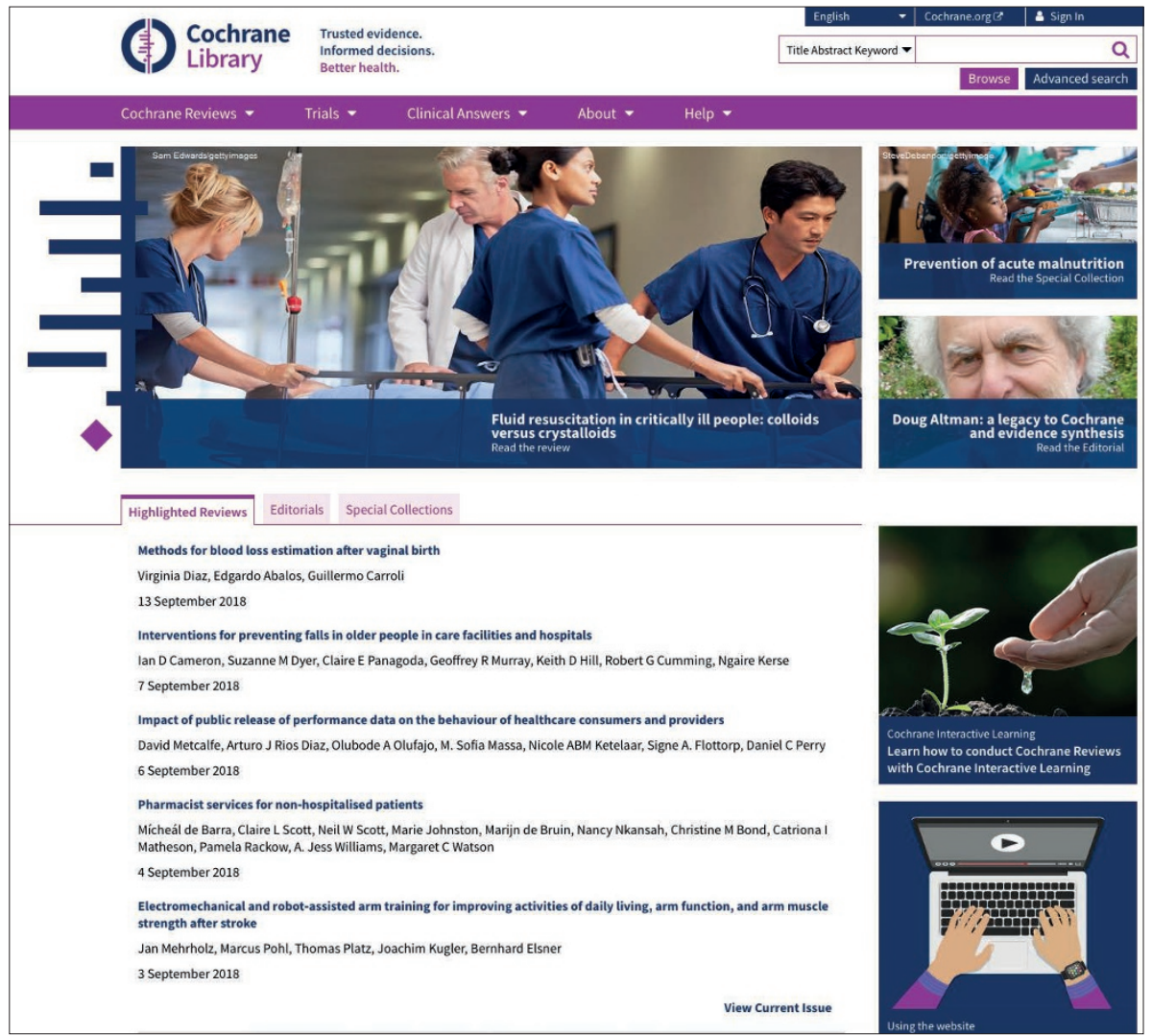

Figure 1. Cochrane Library home page

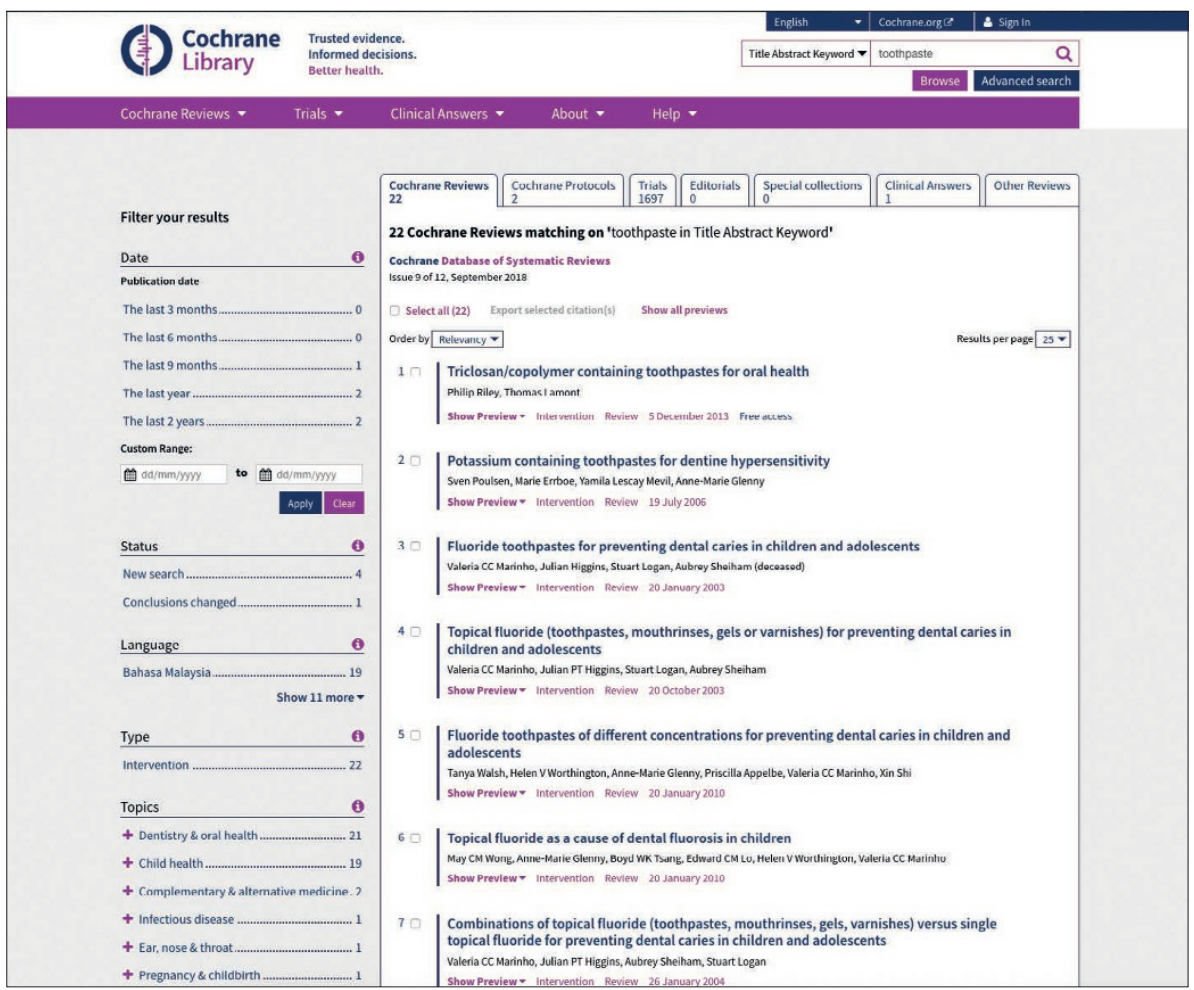

Figure 2. Cochrane Library search page 